\title{
A GEOMETRIC REALISATION OF THE LEPOWSKY BERNSTEIN GELFAND GELFAND RESOLUTION
}

\author{
MICHAEL MURRAY AND JOHN RICE
}

(Communicated by Jonathan M. Rosenberg)

\begin{abstract}
We consider the filtration of the flag manifold arising from the orbits of a parabolic subgroup, and show how its Cousin complex realises Lepowsky's construction of the generalised BGG resolution.
\end{abstract}

\section{INTRODUCTION}

Bernstein, Gelfand, and Gelfand [BGG, 1975] constructed a remarkable resolution by Verma modules for each finite dimensional irreducible representation of a complex, semisimple Lie algebra $\mathfrak{g}$. If $W_{\lambda}$ is the irreducible representation of highest weight $\lambda$ then the resolution has the form

$$
\rightarrow \cdots \rightarrow \bigoplus_{\ell(w)=2} V_{w}(\lambda) \rightarrow \bigoplus_{\ell(w)=1} V_{w}(\lambda) \rightarrow V_{e}(\lambda) \rightarrow W_{\lambda} \rightarrow 0,
$$

where $V_{w}(\lambda)$ is the Verma module of highest weight $w(\lambda+\rho)-\rho, \rho$ is one half the sum of the positive roots, $w$ is an element of the Weyl group $\mathscr{W}$ and $\ell(w)$ is its length.

The arguments of Bernstein, Gelfand, and Gelfand are algebraic and combinatorial, using the structure of the Weyl group to construct the maps in the resolution (1.1) and to show that it is exact. However, $W_{\lambda}$ can be realised by sheaf cohomology on $G / B$, where $B$ is a Borel subgroup, and the Weyl group $\mathscr{W}$ labels the Schubert cells, i.e. the orbits of $B$ on $G / B$, so it is natural to look for a geometric realisation of the BGG resolution in terms of these objects. Kempf (1978) showed that the Cousin complex for the local cohomology of a filtered space produces the dual of the BGG resolution when applied to the filtration of $G / B$ obtained from the closures of the Schubert cells. For $\lambda$ a dominant integral weight, Kempf shows that if $X_{w}$ is the Schubert cell labelled by $w$, that is, the orbit $B w B$, then the dual of the Verma module $V_{w}(\lambda)$ is the algebraic local cohomology group $H_{X_{w}}^{n-\ell(w)}\left(G / B, \mathscr{L}_{\lambda}\right)$, where $\mathscr{L}_{\lambda}$ is the line bundle on $G / B$ associated with $\lambda$.

Notice that $V_{w}(\lambda)$ is a $(\mathfrak{g}, B)$ module, so it is natural that its geometric realisation should involve objects supported on a $B$ orbit in order that $B$ act globally while $G$ acts only infinitesimally.

Received by the editors May 17, 1990 and, in revised form, September 14, 1990.

1980 Mathematics Subject Classification (1985 Revision). Primary 22E46, 22E47, 32C36. 
If $P$ is a parabolic subgroup of $G$ and $V$ a finite dimensional irreducible representation of $\mathfrak{g}$, then Lepowsky (1977) showed that $V$ has a resolution by generalised Verma modules, i.e. modules constructed by inducing from $\mathfrak{p}$ rather than from $\mathfrak{b}$. This resolution has the same form as the BGG resolution but now the $w$ are chosen to be the shortest length coset representatives for the left cosets of $\mathscr{W}_{P}$, the Weyl group of $P$. We shall give a geometric description of his construction.

Because the cosets of $\mathscr{W}_{P}$ label the orbits of $B$ on $G / P$ one might be tempted to look there for a geometric realisation of Lepowsky's resolution. However, explicit calculations on projective space show that this is a false hope. Recalling that the generalised Verma modules are $(\mathfrak{g}, P)$ modules, it is more natural to look for something on which $P$ acts. We shall show that it is the Cousin complex for the $P$ orbits on $G / B$ which realises the Lepowsky BGG resolution.

Any generalised Verma module, being a highest weight module, is a quotient of some Verma module. Lepowsky's construction depends on determining the kernel of the quotient map as a sum of Verma modules. This presentation of a generalised Verma module can be extended to a complete resolution of it by Verma modules, and we give a geometric realisation of this resolution, also as a Cousin complex.

\section{Generalised Verma modules}

Recall (Lepowsky (1977)) that being a highest weight module the generalised Verma module $V_{P}(\mu)$ must be a quotient of the Verma module $V(\mu)$ (note that we are labelling Verma modules by their highest weights). In fact Lepowsky shows that the kernel of

$$
V(\mu) \rightarrow V_{P}(\mu)
$$

is

$$
K_{\mu}=\sum_{\substack{\ell(\sigma)=1 \\ \sigma \in \boldsymbol{W}_{p}}} V_{\sigma}(\mu) .
$$

The Verma module $V(\mu)$ contains all the Verma modules $V_{\sigma}(\mu)$ as submodules and the inclusion relations are the reverse of the ordering on the Weyl group. It is in this sense that we say the kernel is a sum of Verma modules. Notice that the sum is not direct and that it is equally true to say that the sum is over all elements of $\mathscr{W}_{P}$.

These results enable the Lepowsky BGG resolution to be obtained from the BGG resolution as a "quotient." That is, Lepowsky shows that any homomorphism $f: V(\mu) \rightarrow V(\lambda)$ descends to a quotient $f: V_{P}(\mu) \rightarrow V_{P}(\lambda)$ because $f\left(K_{\mu}\right) \subset K_{\lambda}$. Bernstein, Gelfand, and Gelfand construct their resolution by using the inclusions $V(\mu) \subset V(\lambda)$ added together with careful choices of signs to give the maps

$$
\bigoplus_{\ell(w)=i} V_{w}(\lambda) \rightarrow \bigoplus_{\ell(w)=i-1} V_{w}(\lambda)
$$

in the resolution. With the same choices of signs, and with $\mathscr{W}_{P}{ }^{\perp}$ denoting the left coset representatives of $\mathscr{W}_{P}$ of shortest length, the quotient maps define 
maps

$$
\bigoplus_{\substack{\ell(w)=i \\ w \in \mathscr{Y}_{P}^{\perp}}} V_{P w}(\lambda) \rightarrow \bigoplus_{\substack{\ell(w)=i-1 \\ w \in \mathscr{Y}_{P}^{\perp}}} V_{P w}(\lambda)
$$

which give the Lepowsky BGG resolution.

Our point is that the Cousin complex for the $P$ orbits is obtained from that for the $B$ orbits in a similar manner.

\section{THE GEOMETRIC REALISATION}

If $X \supset Z_{0} \supset Z_{1} \supset \cdots \supset Z_{d}$ is a filtration of the topological space $X$ by closed subsets and $\mathscr{F}^{\cdot}$ is a complex of sheaves on $X$, then we obtain a filtered complex

$$
\mathscr{F}_{Z_{0}}(X) \supset \mathscr{F}_{Z_{1}}(X) \supset \cdots \supset \mathscr{F}_{Z_{d}}(X)
$$

where $\mathscr{F}_{Z} \cdot(X)$ denotes the global sections of $\mathscr{F}$ supported in the closed set $Z$. In our case $X$ will be an analytic manifold and the closed sets will be analytic subsets, i.e. those defined locally by the vanishing of holomorphic functions. We deal only with open sets which are the complements of such closed sets, that is, we confine ourselves to the Zariski topology. For any such set $U=X \backslash Z$ and any sheaf $\mathscr{F}$, let $\widehat{\mathscr{F}}(U)$ denote the meromorphic sections of $\mathscr{F}$ over $U$. These are the sections $s$ such that for some fixed integer $n$, and for every $\phi$ in the defining ideal of $Z, \phi^{n} s$ is the restriction to $U$ of a global section. $\widehat{\mathscr{F}}$ is a subsheaf of $\mathscr{F}$, and $\widehat{\mathscr{F}_{Z}}(X)$ consists of sections which locally are annihilated by some power of the defining ideal of $Z$. In Murray and Rice (1990) we considered sheaves $\mathscr{D}$ of currents and showed that the Division Theorem of Malgrange (Malgrange (1959/60)) is equivalent to the assertion that $\widehat{\mathscr{D}}$ is flabby as a sheaf on the Zariski topology. In what follows we shall work only with the "algebraic" subsheaves $\widehat{\mathscr{F}}$, and so we will not distinguish notationally between $\mathscr{F}$ and $\widehat{\mathscr{F}}$.

If $\mathscr{F}^{\circ}$ is a flabby resolution of a sheaf $\mathscr{F}$, then the cohomology of the complex $\mathscr{F}_{Z}(X)$ is (by definition), the algebraic local cohomology of $\mathscr{F}$ along $Z$, which we shall simply call the local cohomology and denote $H_{Z}^{\bullet}(X, \mathscr{F})$. Since for flabby sheaves $\mathscr{F}$ the map $\mathscr{F}(X) \rightarrow \mathscr{F}(U)$ is surjective for any open set $U$, the sequence

$$
0 \rightarrow \mathscr{F}_{Z_{j+1}}(X) \rightarrow \mathscr{F}_{Z_{j}}(X) \rightarrow \mathscr{F}_{Z_{j} \backslash Z_{j+1}}\left(X \backslash Z_{j+1}\right) \rightarrow 0
$$

is exact. Moreover, the map $\mathscr{F}_{Z_{j} \backslash Z_{j+1}}\left(X \backslash Z_{j+1}\right) \rightarrow \mathscr{F}_{Z_{j} \backslash Z_{j+1}}(U)$ is an isomorphism for any open set $U$ in which $Z_{j} \backslash Z_{j+1}$ is closed. The cohomology $H_{Z_{j} \backslash Z_{j+1}}(U, \mathscr{F})$ is therefore the same for any open set containing $Z_{j} \backslash Z_{j+1}$ as a closed set, and we make this the definition of $H_{Z_{j} \backslash Z_{j+1}}(X, \mathscr{F})$.

The mapping cone of $l: \mathscr{F}_{Z_{j+1}}(X) \rightarrow \mathscr{F}_{Z_{j}}(X)$, which we denote $\widehat{\mathscr{F}}_{Z_{j} \backslash Z_{j+1}}(X)$, consists in degree $k$ of pairs $(\alpha, \beta)$ with $\alpha \in \mathscr{F}_{Z_{j+1}}^{k+1}(X)$ and $\beta \in \mathscr{F}_{Z_{j}}^{k}(X)$, and has differential $D(\alpha, \beta)=(d \alpha, l(\alpha)-d \beta)$. The cohomology of $\widehat{\mathscr{F}}_{Z_{j} \backslash Z_{j+1}}(X)$ is naturally isomophic to that of $\mathscr{F}_{Z_{j} \backslash Z_{j+1}}(X)$. In Murray and Rice (1990) we showed that the map $\delta:(\alpha, \beta) \rightarrow(0, \alpha)$ provides a horizontal differential which makes these mapping cone complexes, the $j$ th one shifted by degree $j$, 
into the columns of a double complex with exact rows. By the zig-zag lemma (Bott and Tu (1982)), the cohomology of this double complex is isomorphic to the cohomology of $\mathscr{F}_{Z_{0}}(X)$, i.e. to $H_{Z_{0}}^{\cdot}(X, \mathscr{F})$. The vertical cohomology of the double complex is $H_{Z_{j} \backslash Z_{j+1}}^{k+j}(X, \mathscr{F})$ and the horizontal differential induces the complex

$$
\rightarrow H_{Z_{0} \backslash Z_{1}}^{k}(X, \mathscr{F}) \rightarrow H_{Z_{1} \backslash Z_{2}}^{k+1}(X, \mathscr{F}) \rightarrow H_{Z_{2} \backslash Z_{3}}^{k+2}(X, \mathscr{F}) \rightarrow \cdots
$$

in the $k$ th row. These complexes are called Cousin complexes. If all except the $k$ th Cousin complex is zero, then once again the zig-zag lemma shows that its cohomology is isomorphic to the cohomology of the double complex shifted by $k$ degrees. The double complex therefore plays the role of the Cechde Rham complex, and the isomorphism between $H_{Z_{0}}^{\cdot}(X)$ and the cohomology of the Cousin complex is the direct analogue of the de Rham isomorphism.

Kempf (1978) applies these ideas in the case where $X=G / B$ and $Z_{j}$ is the closure of the orbits of $B$ with codimension greater than or equal to $j$. Then $Z_{j} \backslash Z_{j+1}$ is the union of the $B$ orbits of codimension $j$, that is $X_{w}$ with $\ell(w)=n-j$. Since these are disjoint and open in $Z_{j}$, it is easy to see that

$$
H_{Z_{j} \backslash Z_{j+1}}^{k+j}(X, \mathscr{F})=\bigoplus_{\ell(w)=n-j} H_{X_{w}}^{k+j}(X, \mathscr{F})
$$

The results of Kempf (1978) and Brylinski (1981) show that, for a dominant line bundle $\mathscr{L}_{\lambda}$, one has $H_{X_{w}}^{i}\left(X, \mathscr{L}_{\lambda}\right)=0$ unless $\ell(w)=n-i$, when it is the dual of $V_{w}$. Equally well one can use their results, or the direct arguments of Murray and Rice (1990), to show that if $\mathscr{K}$ is the sheaf of holomorphic $n$-forms then

$$
H_{X_{w}}^{i}\left(X, \mathscr{L}_{\lambda} \otimes \mathscr{K}\right)= \begin{cases}0 & \text { if } \ell(w) \neq n-i \\ V_{w}(\lambda) & \text { if } \ell(w)=n-i\end{cases}
$$

The Cousin complexes are therefore zero except for the one with $k=0$; its terms are clearly those of the BGG resolution. Its cohomology is $H^{*}\left(X, \mathscr{L}_{\lambda} \otimes \mathscr{K}\right)$, since $Z_{0}=X$, and by Bott's theorem this vanishes except in degree $n$, where it is $W_{\lambda}$. This shows that the Cousin complex is a resolution of $W_{\lambda}$ and identifies it with the BGG resolution.

Consider the orbits of $P$ on $G / B$. The minimal $P$ orbit, which lies through the identity coset, can be identified as $P / B=L / L \cap B$, where $L$ is a Levi factor of $P$. Note that $L \cap B$ is a Borel subgroup of $L$, and each $B$ orbit in $P / B$ is actually an $L \cap B$ orbit. Therefore $P / B$ is the union of $B$ orbits $X_{v}$ where $v$ belongs to $\mathscr{W}_{P}$, which is also the Weyl group of $L$ as well as of $P$. The dimension of $P / B$ is the length of the longest element of $\mathscr{W}_{P}$, which we denote by $d$. More generally, each $P$ orbit is a union of $B$ orbits $X_{v}$, where $v$ ranges over a coset of $\mathscr{W}_{P}$ in $\mathscr{W}$, and contains a unique minimal $B$ orbit $X_{w}$ of codimension $d$, where $w$ is consequently the element of this coset with shortest length. Recall that $\mathscr{W}_{P}{ }^{\perp}$ denotes the set of all these shortest length representatives, and let $Y_{w}=P w B$ denote the $P$ orbit labelled by $w \in \mathscr{W}_{P}{ }^{\perp}$, so that $Y_{w}$ is the union of $B$ orbits

$$
Y_{u}=\bigcup_{v \in \mathscr{W}_{P}} X_{v w}
$$


Theorem 1. For each $w \in W_{P}^{\perp}$ and dominant line bundle $\mathscr{L}_{\lambda}$ over $X=G / B$, the cohomology $H_{Y_{w}}^{n-\ell(w)}\left(X, \mathscr{K} \otimes \mathscr{L}_{\lambda}\right)$ is isomorphic to $V_{P w}(\lambda)$.

Proof. Let $U_{w}=X \backslash\left(\bar{Y}_{w} \backslash Y_{w}\right)$ so that $Y_{w}$ is closed in the open set $U_{w}$. Let $r=n-\ell(w)-d$ be the codimension of $Y_{w}$. We consider the filtration

$$
U_{w} \supset Y_{w} \supset Y_{w} \cap Z_{r+1} \supset \cdots \supset Y_{w} \cap Z_{r+d}=X_{w},
$$

where $Z_{j}$ is the closure of the Schubert cells of codimension greater than or equal to $j$, as described for the BGG resolution. Since

$$
Y_{w} \cap\left(Z_{r+k} \backslash Z_{r+k+1}\right)=\bigcup_{\substack{\ell(v)=d-k \\ v \in \mathscr{W}_{P}}} X_{v w},
$$

it follows by the results of Kempf that the Cousin complexes for $\mathscr{K} \otimes \mathscr{L}_{\lambda}$ determined by this filtration are zero except in the $r$ th row, which is

$$
\begin{aligned}
& \rightarrow \bigoplus_{\substack{\ell(v) d \\
v \in \mathscr{\not}_{P}}} H_{X_{v w}}^{r}\left(X, \mathscr{K} \otimes \mathscr{L}_{\lambda}\right) \rightarrow \\
& \cdots \rightarrow \bigoplus_{\substack{\ell(v)=1 \\
v \in \mathscr{W}_{P}}} H_{X_{v w}}^{r+d-1}\left(X, \mathscr{K} \otimes \mathscr{L}_{\lambda}\right) \rightarrow H_{X_{w}}^{r+d}\left(X, \mathscr{K} \otimes \mathscr{L}_{\lambda}\right) \rightarrow 0 .
\end{aligned}
$$

By the theorem of de Rham type which we described above, the cohomology of the complex 3.7 is $H_{Y_{w}}^{r+k}\left(X, \mathscr{K} \otimes \mathscr{L}_{\lambda}\right)$ in degree $k$. In particular, from the end of 3.7 we obtain the exact sequence

$$
\sum_{\substack{\ell(\sigma)=1 \\ \sigma \in \mathscr{P}_{P}}} V_{\sigma w}(\lambda) \rightarrow V_{w}(\lambda) \rightarrow H_{Y_{w}}^{n-\ell(w)}\left(X, \mathscr{K} \otimes \mathscr{L}_{\lambda}\right) \rightarrow 0
$$

and it follows from the result of Lepowsky that $H_{Y_{w}}^{n-\ell(w)}\left(X, \mathscr{K} \otimes \mathscr{L}_{\lambda}\right)$ is $V_{P w}(\lambda)$.

Let $\tilde{Z}_{j}$ be the closure of the union of the $P$-orbits $Y_{w}$ of codimension greater than or equal to $j . \widetilde{Z}_{j} \backslash \widetilde{Z}_{j+1}$ is the disjoint union of the $P$-orbits $Y_{w}$ of codimension $j$, each of which is open in $\tilde{Z}_{j}$. We therefore obtain, for any sheaf $\mathscr{F}$, the Cousin complexes

$$
\begin{aligned}
\rightarrow & \bigoplus_{\substack{(w)=n-d \\
w \in \mathscr{\vartheta}_{P}^{\perp}}} H_{Y_{w}}^{k}(X, \mathscr{F}) \rightarrow \\
& \cdots \rightarrow \bigoplus_{\substack{\left\{(w)=1 \\
w \in \boldsymbol{\eta}_{P}^{\perp}\right.}} H_{Y_{w}}^{k+n-d-1}(X, \mathscr{F}) \rightarrow H_{Y_{e}}^{k+n-d}(X, \mathscr{F}) \rightarrow 0 .
\end{aligned}
$$

For $\mathscr{F}=\mathscr{K} \otimes \mathscr{L}_{\lambda}$, Theorem 1 shows that the terms of the Cousin complex 3.8, with $k=d$, are the generalised Verma modules of Lepowsky's resolution. If this were the only nonzero Cousin complex, then the Cousin double complex would compute the cohomology of $\mathscr{K} \otimes \mathscr{L}_{\lambda}$, and the same argument as for the standard BGG resolution would identify 3.8 in the case $k=d$ with Lepowsky's generalised BGG resolution. Such an argument can be made using the $D$-module cohomology vanishing theorems of Beilinson and Bernstein (1981). However, the results of Lepowsky cited in $\S 2$ allow a more elementary proof. 
Indeed the map from $V_{w}(\lambda)$ to $V_{P w}(\lambda)$ corresponds to the natural map between local cohomology groups, and diagrams such as

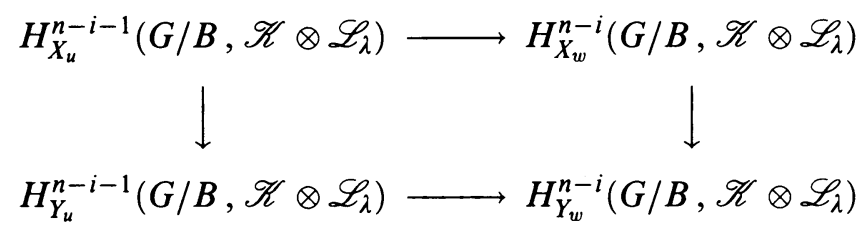

all commute. Hence we have

Theorem 2. The Cousin complex

$$
\begin{aligned}
\rightarrow \bigoplus_{\substack{\ell(\sigma)=1 \\
\sigma \in \boldsymbol{Y}_{P}}} H_{Y_{w}}^{k+1}(X, \mathscr{F}) \rightarrow \cdots \rightarrow \bigoplus_{\substack{\ell(\sigma)=-d-1 \\
\sigma \in \boldsymbol{\vartheta}_{P}}} H_{Y_{w}}^{k+n-d-1}(X, \mathscr{F}) \\
\rightarrow \bigoplus_{\substack{\ell(\sigma)=n-d \\
\sigma \in \boldsymbol{Y}_{P}}} H_{Y_{w}}^{k+n-d}(X, \mathscr{F}) \rightarrow 0
\end{aligned}
$$

is the Lepowsky Bernstein Gelfand Gelfand resolution.

We can also give a direct proof of the local cohomology vanishing theorem.

Theorem 3. For a dominant line bundle $\mathscr{L}_{\lambda}$

$$
H_{Y_{w}}^{i}\left(X, \mathscr{K} \otimes \mathscr{L}_{\lambda}\right)=0
$$

if $i \neq n-\ell(w)$.

Proof. The complex 3.7 is a complex of Verma modules which is "part" of the BGG resolution. To be more precise recall the construction of the BGG resolution in Bernstein, Gelfand, and Gelfand (1975). They regard all the Verma modules $V_{w}$ as being included in $V_{e}$. Then they show that there is a special function $s\left(w_{1}, w_{2}\right)$ defined for any two Weyl group elements $w_{1}, w_{2}$ with $\ell\left(w_{1}\right)=\ell\left(w_{2}\right)+1$ and $w_{1}=\sigma_{\alpha} w_{2}$, for some simple root reflection $\sigma_{\alpha}$ and taking the values plus or minus one. The maps

$$
\bigoplus_{\ell(w)=i} V_{w} \rightarrow \bigoplus_{\ell(w)=i-1} V_{w}
$$

defining the BGG resolution are obtained by adding together the inclusions of $V_{w_{1}}$ into $V_{w_{2}}$ multiplied by the sign $s\left(w_{1}, w_{2}\right)$. If we restrict to a Levi subgroup $L$ of $G$, then we can restrict the function $s$ to $\mathscr{W}_{P}$, the Weyl group of $L$. The irreducible $L$-module $W_{\lambda}$ has a BGG resolution by Verma modules for $L$ and if we extend these Verma modules to $\mathscr{U}(\mathfrak{p})$ modules by making the nilpotent part of $\mathfrak{p}$ act trivially, then we can tensor by $\mathscr{U}(\mathfrak{g})$ over $\mathscr{U}(\mathfrak{p})$ to obtain $\mathscr{U}(\mathfrak{g})$ modules. It is straightforward to check that these are the generalised Verma modules for $\mathfrak{g}$ with the corresponding highest weight and that this operation respects exactness. It follows that the complex

$$
\rightarrow \cdots \rightarrow \bigoplus_{\substack{\ell(v)=2 \\ v \in \boldsymbol{V}_{p}}} V_{v w}(\lambda) \rightarrow \bigoplus_{\substack{\ell(v)=1 \\ v \in \boldsymbol{\varkappa}_{p}}} V_{v w}(\lambda) \rightarrow V_{w}(\lambda) \rightarrow V_{P w}(\lambda) \rightarrow 0
$$

is exact and this is the same as the complex 3.7. Consequently the only nonzero local cohomology $H_{Y_{w}}^{i}\left(X, \mathscr{K} \otimes \mathscr{L}_{\lambda}\right)$ is in dimension $n-\ell(w)$.

Corollary. The Cousin complex 3.7 is a resolution of $H_{Y_{w}}^{n-\ell(w)}\left(X, \mathscr{K} \otimes \mathscr{L}_{\lambda}\right)$. 


\section{REFERENCES}

I. N. Bernstein, I. M. Gelfand, and S. I. Gelfand (1975), Differential operators on the base affine space and a study of $\mathfrak{g}$-modules, Lie Groups and Their Representations. Summer School of the Bolyai János Math. Soc. (I. M. Gelfand, editor), Halsted Press, New York, pp. 21-64.

J. L. Brylinski (1981), Differential operators on the flag varieties, Astérisque 87-88, 43-60.

A. Beilinson and J. Bernstein (1981), Localisation de $\mathfrak{g}$ modules, C. R. Acad. Sci. Paris Ser. I Math. 292, 15-18.

R. Bott and L. Tu (1982), Differential forms in algebraic geometry, Graduate Texts in Math., Springer, New York, Heidelberg and Berlin.

P. Griffiths and J. Harris (1978), Principles of algebraic geometry, Intersci. Publ., New York.

G. Kempf (1978), The Grothendieck-Cousin complex of an induced representation, Adv. in Math. 29, 310-396.

J. Lepowsky (1977), A generalization of the Bernstein Gelfand Gelfand resolution, J. Algebra 49, 469-511.

B. Malgrange (1959/60), Division des distributions, Séminaire Schwartz, $4^{e}$ année, Part 3, Articles 21-25, Faculté des Sciences de Paris.

M. K. Murray and J. W. Rice (1990), Dolbeault and Cousin-Dolbeault theorems for algebraic local cohomology, preprint.

Mathematics Research Section, School of Mathematical Sciences, ias, Australian National University, GPO Box 4, CanberRa, ACT 2601, Australia

School of Information Science and Technology, Flinders University of South Australia, GPO Box 2100, Adelaide, SA 5001, Australia 